\title{
Interior of a unitarily evaporating black hole
}

\author{
Yasunori Nomura ${ }^{\circ}$ \\ Berkeley Center for Theoretical Physics, Department of Physics, University of California, Berkeley, \\ California 94720, USA; Theoretical Physics Group, Lawrence Berkeley National Laboratory, Berkeley, \\ California 94720, USA; and Kavli Institute for the Physics and Mathematics of the Universe (WPI), \\ UTIAS, The University of Tokyo, Kashiwa, Chiba 277-8583, Japan
}

(Received 16 December 2019; accepted 15 June 2020; published 1 July 2020)

\begin{abstract}
We study microscopic operators describing the experience of an observer falling into the horizon of a unitarily evaporating black hole. For a young black hole, these operators can be taken to act only on the degrees of freedom in the black hole region: the soft—or stretched horizon-modes as well as the semiclassical modes in the zone region. On the other hand, for an old black hole, the operators must also involve radiation emitted earlier; the difference between the two cases comes from statistics associated with the coarse graining performed to obtain the effective theory of the interior. We find that the operators relevant for the interior theory can be defined globally as standard linear operators throughout the microstates, which obey the correct algebra up to corrections exponentially suppressed in the ratio of excitation energy to the Hawking temperature. We conjecture that the existence of such global operators is required for the emergence of the semiclassical picture. We also elucidate the relation between the present construction and entanglement wedge reconstruction of the interior.
\end{abstract}

DOI: 10.1103/PhysRevD.102.026001

\section{INTRODUCTION}

Since the discovery of the thermodynamics of a black hole [1,2], studying its physics has been driving our understanding of spacetime and gravity at the fundamental level. In particular, addressing the apparent contradiction [3] between the thermodynamic aspects of a black hole and the principles of quantum mechanics has led to many important ideas, including black hole complementarity [4]. While we have found that the principles of quantum mechanics prevail in the end $[5,6]$, paradoxes still remain. One of the latest forms of these is the difficulty of reconciling unitary evolution of a black hole with the existence of its interior [7].

The purpose of this paper is to study how operators describing the interior of a unitarily evaporating black hole can be constructed at the microscopic level. For a black hole in asymptotically flat spacetime [or a small black hole in anti-de Sitter (AdS) spacetime], we work in the framework of Refs. [8,9]. This framework describes a black hole as a state in which the hard modes - the modes relevant for describing small objects around the black hole-are entangled in a generic manner with the soft modes-the

Published by the American Physical Society under the terms of the Creative Commons Attribution 4.0 International license. Further distribution of this work must maintain attribution to the author(s) and the published article's title, journal citation, and DOI. Funded by SCOAP ${ }^{3}$. degrees of freedom comprising the majority of the black hole. This entanglement is generated by a strong, chaotic dynamics near the horizon. We will also discuss an extension of the framework to a large AdS black hole.

A black hole forms when the system-specific properties, e.g., the details of the surface of a collapsing star, are strongly redshifted due to gravity, so that the system appears to be in a vacuum state at the semiclassical level. This redshift makes the majority of the degrees of freedom unobservable for a long time. These "hidden" degrees of freedom, associated with the black hole, have an exponentially large density of states [1] and are distributed mostly near the horizon. While they are very soft when measured in the asymptotic region, their intrinsic dynamical scale is larger near the horizon due to gravitational blueshift, reaching the string scale at the stretched horizon [4]. The dynamics in this region is chaotic [10,11] across all low-energy species, giving generic entanglement between the hidden degrees of freedom (soft modes) and the others which can be used to describe small excitations around the black hole (hard modes). In fact, this entanglement is the origin [8] of the thermality of the black hole [2].

While the strong dynamics near the stretched horizon cannot be described by a low-energy quantum field theory, we expect that it is unitary, as implied by the AdS/CFT correspondence [6]. The fact that this dynamics lies outside the validity of the low-energy theory allows for avoiding the conclusion of information loss [3] reached by semiclassical calculation. The picture of the black hole interior 
can emerge through a coarse graining of the degrees of freedom that cannot be physically resolved by an infalling observer, whose consistency with black hole's unitary evolution is ensured by a specific entanglement structure between the hard modes, soft modes, and early radiation [9].

In this paper, we analyze how the operators in the coarsegrained, effective theory of the interior can be realized in the original microscopic theory. We show that the construction of these operators is not unique. In particular, we find that the operators can be written without involving an element outside the black hole region for a young black hole, a black hole that is not yet maximally entangled with the early radiation. On the other hand, for an old black hole, radiation degrees of freedom must be involved, despite the fact that the degrees of freedom describing a falling object are not directly entangled with the early radiation. We study how this happens both in the cases of a black hole in asymptotically flat spacetime and a large black hole in AdS spacetime. This analysis elucidates which features of the analyses using a large AdS black hole may apply to a black hole in asymptotically flat spacetime.

The construction described in this paper addresses all the issues raised regarding the interior of a unitarily evaporating black hole [7,12-15]. In particular, we find that all the operators relevant for the effective theory of the interior can be represented as standard linear quantum operators at the microscopic level, which obey the correct algebra up to corrections exponentially suppressed in the ratio of excitation energy to the Hawking temperature, which is typically a very large number. We also argue that the construction preserves spacetime locality at the semiclassical level.

The organization of this paper is as follows. In Sec. II, we describe the framework of Refs. [8,9], highlighting features relevant for our discussion. In Sec. III, we present our analysis of interior operators for a black hole in asymptotically flat spacetime (or a small black hole in AdS spacetime). We construct operators that play the role of annihilation and creation operators in the effective theory of the interior erected at a given time in a distant description. We find that for a young black hole, these operators can be chosen to act only on the hard and soft modes, while for an old black hole, they must also involve early radiation.

In Sec. IV, which was added after the first version of this paper was posted, in response to an intriguing paper by Penington, Shenker, Stanford, and Yang [16], we perform a similar analysis for a large AdS black hole. In this case, we obtain a result resembling that of Ref. [16]: for an old black hole, we can construct certain operators analogous to the interior ones which act only on early radiation. These operators, however, are not relevant for constructing the effective interior theory erected at that time; as in the case of a flat space black hole, operators relevant for such an effective theory must involve both soft modes and early radiation. A key to reconcile these two results and figuring out the relation between our construction and entanglement wedge reconstruction of the interior [17-19] is time evolution, which we will discuss.

In Sec. V, we conclude with a discussion on the relation between our findings and the emergence of semiclassical spacetime. We conjecture that the existence of (approximately) global operators is required for the emergence of the semiclassical picture.

Throughout the paper, we focus on black holes in fourdimensional spacetime that are not significantly rotating or charged, although the restriction on specific spacetime dimensions or on nonrotating, noncharged black holes is not essential. We adopt natural units $c=\hbar=1$, and $l_{\mathrm{P}}$ denotes the Planck length.

\section{FLAT SPACE BLACK HOLE IN A DISTANT DESCRIPTION}

In this section and the next, we discuss a flat space (or a small AdS) black hole using the framework of Refs. [8,9]. A key feature of the framework is that the thermal nature of a black hole in a distant description can be viewed as arising from entanglement between hard and soft modes of lowenergy quantum fields. ${ }^{1}$ Modes of a low-energy quantum field in the zone region (also called the thermal atmosphere)

$$
r_{s} \leq r \leq r_{z}
$$

are decomposed into hard and soft modes; the hard modes have frequencies $\omega$ and gaps among them $\Delta \omega$ larger than

$$
\Delta \approx O\left(\frac{1}{M l_{\mathrm{P}}^{2}}\right)
$$

as measured in the asymptotic region, while the soft modes have $\omega \lesssim \Delta$. (The hard modes are those which the bulk theory can discriminate individually at the quantum level.) Here, $r_{\mathrm{z}} \approx 3 M l_{\mathrm{P}}^{2}$, and $r_{s}$ is the location of the stretched horizon, given by

$$
r_{s}-2 M l_{\mathrm{P}}^{2} \sim \frac{l_{\mathrm{S}}^{2}}{M l_{\mathrm{P}}^{2}} .
$$

In a distant description, the classical spacetime picture is applicable only outside the stretched horizon, and its location is determined by the condition that the proper distance from the mathematical horizon, $r=2 M l_{\mathrm{P}}^{2}$, is of order the string length $l_{s}$.

While the frequencies of the soft modes are small as measured in the asymptotic region, their intrinsic dynamical scale is larger at a location deeper in the zone, due to large gravitational blueshift. In particular, it is of order the string scale near the stretched horizon, where a majority of

\footnotetext{
${ }^{1}$ Here and below, low-energy fields mean quantum fields existing below the string scale $1 / l_{s}$.
} 
the modes reside. (The distribution of the soft modes is given by the entropy density that goes as the cubic power of the blueshift factor $1 / \sqrt{1-2 M l_{\mathrm{P}}^{2} / r}$.) The dynamics of the soft modes there, therefore, cannot be described by the low-energy theory. ${ }^{2}$ It is this dynamics that is responsible for unitarity of the Hawking emission process.

The quantity $\Delta$ in Eq. (2) is naturally taken to be somewhat, e.g., by a factor of $O(10)$, larger than the Hawking temperature

$$
T_{\mathrm{H}}=\frac{1}{8 \pi M l_{\mathrm{P}}^{2}} .
$$

Since $\Delta$ is the inverse timescale for single Hawking emission, the uncertainty principle prevents us from specifying the energy of the black hole better than that. Below, we will assume that the energy (mass) of a black hole is determined with this maximal precision. A superposition of black holes of masses differing more than $\Delta$ can be treated in a straightforward manner.

At a given time $t$, the state of the entire system-with the black hole being put in the semiclassical vacuum state-is given by

$$
|\Psi(M)\rangle=\sum_{n} \sum_{i_{n}=1}^{e^{S_{\mathrm{bh}}\left(M-E_{n}\right)}} \sum_{a=1}^{e^{S_{\mathrm{rad}}}} c_{n i_{n} a}\left|\left\{n_{\alpha}\right\}\right\rangle\left|\psi_{i_{n}}^{(n)}\right\rangle\left|\phi_{a}\right\rangle .
$$

Excitations on a black hole background will be discussed later. In this expression, $\left|\left\{n_{\alpha}\right\}\right\rangle$ are orthonormal states of the hard modes, with $n \equiv\left\{n_{\alpha}\right\}$ representing the set of all occupation numbers $n_{\alpha}(\geq 0)$. The index $\alpha$ collectively denotes the species, frequency, and angular-momentum quantum numbers of a mode, and $E_{n}$ is the energy of the state $\left|\left\{n_{\alpha}\right\}\right\rangle$ as measured in the asymptotic region (with precision $\Delta$ ).

$\left|\psi_{i_{n}}^{(n)}\right\rangle$ are orthonormal states of the soft modes entangled with $\left|\left\{n_{\alpha}\right\}\right\rangle$ (and hence having energy $M-E_{n}$ with precision $\Delta$ ). The density of states for the soft modes is given by the Bekenstein-Hawking formula

$$
S_{\mathrm{bh}}(M)=4 \pi M^{2} l_{\mathrm{P}}^{2}
$$

Here, we have assumed that the density of hard-mode states is negligible compared with that of the soft modes. This implies that $i_{n}$ runs over

$$
i_{n}=1, \ldots, e^{S_{\mathrm{bh}}\left(M-E_{n}\right)} .
$$

\footnotetext{
${ }^{2}$ Given that the dynamics is not dictated by the low-energy theory, we may call these modes the stretched horizon degrees of freedom instead of soft modes of low-energy fields near the stretched horizon. In fact, the internal dynamics of these modes are expected to be nonlocal in the spatial directions along the horizon $[10,11]$.
}

Note that with this assumption, the total entropy of the black hole is

$$
\ln \left[\sum_{n} e^{S_{\mathrm{bh}}\left(M-E_{n}\right)}\right] \approx \frac{\mathcal{A}(M)}{4 l_{\mathrm{P}}^{2}},
$$

where $\mathcal{A}(M)=16 \pi M^{2} l_{\mathrm{P}}^{4}$ is the area of the black hole, reproducing the standard interpretation of the BekensteinHawking entropy. The last factor $\left|\phi_{a}\right\rangle$ in Eq. (5) represents the set of orthonormal states representing the system in the far region $r>r_{\mathrm{z}}$.

By the black hole vacuum, we mean that there is no physical excitation identifiable at the semiclassical level. This implies that any attribute a hard-mode state may have is compensated by that of the corresponding soft-mode states (within the precision allowed by the uncertainty principle). In particular, this implies that soft-mode states associated with different hard-mode states are orthogonal:

$$
\left\langle\psi_{i_{m}}^{(m)} \mid \psi_{j_{n}}^{(n)}\right\rangle=\delta_{m n} \delta_{i_{m} j_{n}} .
$$

We also take the states in the far region, $\left|\phi_{a}\right\rangle$, to be given by those of Hawking radiation emitted earlier, i.e., emitted from $r \approx r_{\mathrm{z}}$ to the asymptotic region before time $t . S_{\text {rad }}$ in Eq. (5) is then the coarse-grained entropy of this early radiation.

We take the state in Eq. (5) to be normalized:

$$
\sum_{n} \sum_{i_{n}=1}^{e^{S_{\mathrm{hh}}\left(M-E_{n}\right)}} \sum_{a=1}^{e^{S_{\mathrm{rad}}}}\left|c_{n i_{n} a}\right|^{2}=1
$$

We also assume that the ultraviolet dynamics near the stretched horizon is chaotic, well scrambling the black hole state $[10,11]$. In particular, we assume that the coefficients $c_{n i_{n} a}$ take generic values in the spaces of the hard and soft modes. This implies that statistically

$$
\left|c_{n i_{n} a}\right| \sim \frac{1}{\sqrt{S_{\mathrm{tot}}}}
$$

where

$S_{\mathrm{tot}} \equiv\left(\sum_{n} e^{S_{\mathrm{bh}}\left(M-E_{n}\right)}\right) e^{S_{\mathrm{rad}}}=\left(\sum_{n} e^{-E_{n} / T_{\mathrm{H}}}\right) e^{S_{\mathrm{bh}}(M)} e^{S_{\mathrm{rad}}}$

The standard thermal nature of the black hole is then obtained upon tracing out the soft modes:

$$
\begin{aligned}
\operatorname{Tr}_{\text {soft }}|\Psi(M)\rangle\langle\Psi(M)| & =\frac{1}{\sum_{m} e^{-E_{m} / T_{\mathrm{H}}}} \\
& \times \sum_{n} e^{-E_{n} / T_{\mathrm{H}}}\left|\left\{n_{\alpha}\right\}\right\rangle\left\langle\left\{n_{\alpha}\right\}\right| \otimes \rho_{\phi, n},
\end{aligned}
$$


where $\rho_{\phi, n}$ are reduced density matrices for the early radiation, whose $n$ dependence is small and of order $1 / \sqrt{e^{S_{\text {bh }}(M)}}$. Note that in order to obtain the correct Boltzmann factor, $\propto e^{-E_{n} / T_{\mathrm{H}}}$, it is essential that the coefficients $c_{n i_{n} a}$ take generic values across all low-energy species, i.e., $n$ runs over all low-energy species [9].

In a distant description, the system of a black hole and radiation evolves unitarily with the state taking the form of Eq. (5) at each moment in time. In particular, the entanglement entropy between the black hole and radiation

$$
S_{\text {hard }+ \text { soft }}^{\mathrm{vN}}=S_{\text {rad }}^{\mathrm{vN}}
$$

follows the Page curve [20], where $S_{A}^{\mathrm{VN}}$ is the von Neumann entropy of subsystem $A$. Throughout the history of the black hole, the number of hard modes is much smaller than that of the soft modes. (Note that we are only interested in states that do not yield significant backreaction on spacetime, which limits the number of possible hard-mode states.) Furthermore, the coarse-grained entropies of the soft modes and radiation are both of order $M^{2} l_{\mathrm{P}}^{2}$, except for the very beginning and end of the black hole evolution. We therefore have

$$
\ln \operatorname{dim} \mathcal{H}_{\text {hard }} \ll S_{\text {bh }}\left(M-E_{n}\right), S_{\text {rad }} \approx O\left(M^{2} l_{\mathrm{P}}^{2}\right) .
$$

We stress that this relation holds both before and after the Page time, at which the coarse-grained entropy of the radiation becomes approximately equal to that of the black hole.

Incidentally, by performing the Schmidt decomposition in the space of soft-mode and radiation states for each $n$, the state in Eq. (5) can be written as

$$
|\Psi(M)\rangle=\sum_{n} \sum_{i_{n}=1}^{\mathcal{N}_{n}} c_{i_{n}}^{n}\left|H_{n}\right\rangle\left|S_{n, i_{n}}\right\rangle\left|R_{n, i_{n}}\right\rangle,
$$

where $\left|H_{n}\right\rangle,\left|S_{n, i_{n}}\right\rangle$, and $\left|R_{n, i_{n}}\right\rangle$ are states of the hard modes, soft modes, and radiation, respectively, and

$$
\mathcal{N}_{n}=\min \left\{e^{S_{\mathrm{bh}}\left(M-E_{n}\right)}, e^{S_{\mathrm{rad}}}\right\} .
$$

This expression elucidates why the entanglement argument for firewalls [7] does not apply here. The entanglement responsible for unitarity has to do with the summations of indices $i_{n}$ (in fact, predominantly the vacuum index $i_{0}$ ) shared between the soft-mode and radiation states, while the entanglement necessary for the interior spacetime (see below) has to do with the index $n$. These two are compatible because the number of terms associated with the sum over $n$ is much smaller than $\mathcal{N}_{n}$.

Let us now discuss excitations. A small excitation composed of constituents with $\omega \gtrsim \Delta$ in the zone can be described by annihilation and creation operators acting on the hard modes

$$
\begin{gathered}
b_{\gamma}=\sum_{n} \sqrt{n_{\gamma}}\left|\left\{n_{\alpha}-\delta_{\alpha \gamma}\right\}\right\rangle\left\langle\left\{n_{\alpha}\right\}\right|, \\
b_{\gamma}^{\dagger}=\sum_{n} \sqrt{n_{\gamma}+1}\left|\left\{n_{\alpha}+\delta_{\alpha \gamma}\right\}\right\rangle\left\langle\left\{n_{\alpha}\right\}\right| .
\end{gathered}
$$

In particular, a small object falling from the far region into the black hole, which has the characteristic size $d$ in the angular directions much smaller than the horizon, i.e., $d \ll M l_{\mathrm{P}}^{2}$, can be described in this manner.

In a distant description, a small object falling into the black hole is absorbed into the stretched horizon when it reaches there, whose information will be later sent back to ambient space by Hawking emission. This description, however, is not useful for addressing the question of what the falling object will actually see. Because of the large discrepancy between the stationary frame and the frame of the object, macroscopic time experienced by the object is mapped to an extremely short time for a stationary observer at the location of the object. In particular, anything the object experiences inside the horizon occurs almost "instantaneously" for a stationary observer at $r=r_{s}$. Understanding an object's experiences, therefore, requires time evolution different from the distant one, specifically an evolution associated with the proper time of the object.

\section{EFFECTIVE THEORY OF THE BLACK HOLE INTERIOR}

The effective theory describing the black hole interior can be erected at each time $t$ by coarse graining the soft modes and radiation: the degrees of freedom that cannot be resolved by a fallen object in the timescale available to it. Suppose that the state of the system at time $t$ (with the black hole put in the semiclassical vacuum) is given by Eq. (5) in a distant description. We can then define a set of coarsegrained states each of which is entangled with a specific hard-mode state:

$$
\left.\left.\|\left\{n_{\alpha}\right\}\right\rangle\right\rangle \propto \sum_{i_{n}=1}^{e^{S_{\mathrm{bh}}\left(M-E_{n}\right)}} \sum_{a=1}^{e_{\mathrm{rad}}} c_{n i_{n} a}\left|\psi_{i_{n}}^{(n)}\right\rangle\left|\phi_{a}\right\rangle,
$$

where we have used the same label as the corresponding hard-mode state to specify the coarse-grained state, which we denote by the double ket symbol. Note that the meaning of the coarse graining here is that the state $\left.\left.\|\left\{n_{\alpha}\right\}\right\rangle\right\rangle$ on the left-hand side corresponds to multiple different microstates on the right-hand side, depending on the state of the black hole and radiation represented by the coefficients $c_{n i_{n} a}$.

Using Eq. (11), we find that the squared norm of the (non-normalized) state on the right-hand side of Eq. (20) is given by 


$$
\begin{aligned}
\sum_{i_{n}=1}^{e^{S_{\mathrm{bh}}\left(M-E_{n}\right)}} \sum_{a=1}^{e^{S_{\mathrm{rad}}}}\left|c_{n i_{n} a}\right|^{2} & =\frac{e^{-E_{n} / T_{\mathrm{H}}}}{\left(\sum_{m} e^{-E_{m} / T_{\mathrm{H}}}\right)} \\
& \times\left[1+O\left(\frac{1}{\sqrt{e^{S_{\mathrm{bh}}\left(M-E_{n}\right)} e^{S_{\mathrm{rad}}}}}\right)\right]
\end{aligned}
$$

for generic black hole and radiation microstates. Here, the second term in the square brackets represents the size of statistical fluctuations over different microstates. Therefore, the normalized coarse-grained state $\left.\left.\|\left\{n_{\alpha}\right\}\right\rangle\right\rangle$ is given for generic microstates by

$$
\begin{aligned}
\left.\|\left\{n_{\alpha}\right\}\right\rangle= & e^{E_{n} / 2 T_{\mathrm{H}}} \sqrt{\sum_{m} e^{-E_{m} / T_{\mathrm{H}}}} \\
& \times \sum_{i_{n}=1}^{e^{S_{\mathrm{bh}}\left(M-E_{n}\right)}} \sum_{a=1}^{S_{\mathrm{rad}}} c_{n i_{n} a}\left|\psi_{i_{n}}^{(n)}\right\rangle\left|\phi_{a}\right\rangle,
\end{aligned}
$$

up to a fractional correction of order $1 / e^{\# M^{2} l_{\mathrm{P}}^{2}}$ in the overall normalization, where \# is a number that does not depend on $M l_{\mathrm{P}}$.

The state in the effective theory corresponding to the state in Eq. (5) can be written in terms of the coarse-grained states in Eq. (22) as

$$
\left.\left.\| \Psi(M)\rangle\rangle=\frac{1}{\sqrt{\sum_{m} e^{-E_{m} / T_{\mathrm{H}}}}} \sum_{n} e^{-E_{n} / 2 T_{\mathrm{H}}}\left|\left\{n_{\alpha}\right\}\right\rangle \|\left\{n_{\alpha}\right\}\right\rangle\right\rangle,
$$

regardless of the values of $c_{n i_{n} a}$. This takes the form of the standard thermofield double state in the two-sided black hole picture [21,22], although $\left|\left\{n_{\alpha}\right\}\right\rangle$ here represent the states only of the hard modes. We emphasize that in order to obtain the correct Boltzmann-weight coefficients, $\propto e^{-E_{n} / 2 T_{\mathrm{H}}}$, it is important that the black hole has soft modes with the density of states given by $e^{S_{\mathrm{bh}}\left(E_{\mathrm{soft}}\right)}$ and that the hard and soft modes are well scrambled, giving $c_{n i_{n} a}$ that take values statistically independent of $n$. This coarse graining leads to the apparent uniqueness of the infalling vacuum, despite the existence of exponentially many black hole microstates.

One can now define the annihilation and creation operators $\tilde{b}_{\gamma}$ and $\tilde{b}_{\gamma}^{\dagger}$ acting on the coarse-grained states as

$$
\begin{gathered}
\left.\left.\tilde{b}_{\gamma}=\sum_{n} \sqrt{n_{\gamma}} \|\left\{n_{\alpha}-\delta_{\alpha \gamma}\right\}\right\rangle\right\rangle\left\langle\left\langle\left\{n_{\alpha}\right\} \|,\right.\right. \\
\left.\left.\tilde{b}_{\gamma}^{\dagger}=\sum_{n} \sqrt{n_{\gamma}+1} \|\left\{n_{\alpha}+\delta_{\alpha \gamma}\right\}\right\rangle\right\rangle\left\langle\left\{n_{\alpha}\right\} \|\right.
\end{gathered}
$$

at the level of the effective theory. The annihilation and creation operators relevant for an infalling observer can then be given by

$$
\begin{aligned}
& a_{\xi}=\sum_{\gamma}\left(\alpha_{\xi \gamma} b_{\gamma}+\beta_{\xi \gamma} b_{\gamma}^{\dagger}+\zeta_{\xi \gamma} \tilde{b}_{\gamma}+\eta_{\xi \gamma} \tilde{b}_{\gamma}^{\dagger}\right), \\
& a_{\xi}^{\dagger}=\sum_{\gamma}\left(\beta_{\xi \gamma}^{*} b_{\gamma}+\alpha_{\xi \gamma}^{*} b_{\gamma}^{\dagger}+\eta_{\xi \gamma}^{*} \tilde{b}_{\gamma}+\zeta_{\xi \gamma}^{*} \tilde{b}_{\gamma}^{\dagger}\right),
\end{aligned}
$$

where $b_{\gamma}$ and $b_{\gamma}^{\dagger}$ are the operators in Eqs. (18) and (19), $\xi$ is the label in which the frequency $\omega$ with respect to $t$ is traded with the frequency $\Omega$ associated with the infalling time, and $\alpha_{\xi \gamma}, \beta_{\xi \gamma}, \zeta_{\xi \gamma}$, and $\eta_{\xi \gamma}$ are the Bogoliubov coefficients calculable using the standard field theory method. The generator of time evolution in the infalling description is then given by

$$
H=\sum_{\xi} \Omega a_{\xi}^{\dagger} a_{\xi}+H_{\mathrm{int}}\left(a_{\xi}, a_{\xi}^{\dagger}\right) .
$$

This leads to the physics of a smooth horizon. The existence of the operators $a_{\xi}$ and $a_{\xi}^{\dagger}$ implies that there is a subsector in the original microscopic theory encoding the experience of an object after it crosses the horizon.

How can the operators $\tilde{b}_{\gamma}$ and $\tilde{b}_{\gamma}^{\dagger}$ be constructed at the microscopic level? One way is simply to use Eq. (22) in the expression in Eqs. (24) and (25):

$$
\begin{aligned}
& \tilde{b}_{\gamma}=\left(\sum_{m} e^{-E_{m} / T_{\mathrm{H}}}\right) \sum_{n} \sqrt{n_{\gamma}} e^{\left(E_{n_{-}}+E_{n}\right) / 2 T_{\mathrm{H}}} \\
& \times \sum_{i_{n_{-}}=1}^{e^{S_{\mathrm{bh}}\left(M-E_{n_{-}}\right)}} \sum_{j_{n}=1}^{e^{S_{\mathrm{bh}}\left(M-E_{n}\right)}} \sum_{a=1}^{e^{S_{\mathrm{rad}}}} \sum_{b=1}^{S_{\mathrm{rad}}} c_{n_{-} i_{n_{-}} a} c_{n j_{n} b}^{*} \\
& \times\left|\psi_{i_{n_{-}}}^{\left(n_{-}\right)}\right\rangle\left|\phi_{a}\right\rangle\left\langle\psi_{j_{n}}^{(n)}\right|\left\langle\phi_{b}\right|, \\
& \tilde{b}_{\gamma}^{\dagger}=\left(\sum_{m} e^{-E_{m} / T_{\mathrm{H}}}\right) \sum_{n} \sqrt{n_{\gamma}+1} e^{\left(E_{n_{+}}+E_{n}\right) / 2 T_{\mathrm{H}}} \\
& \times \sum_{i_{n_{+}}=1}^{e^{S_{\mathrm{bh}}\left(M-E_{n_{+}}\right)}} \sum_{j_{n}=1}^{e^{S_{\mathrm{bh}}\left(M-E_{n}\right)}} \sum_{a=1}^{e^{S_{\mathrm{rad}}}} \sum_{b=1}^{e^{S_{\mathrm{rad}}}} c_{n_{+} i_{n_{+}} a} c_{n j_{n} b}^{*} \\
& \times\left|\psi_{i_{n_{+}}}^{\left(n_{+}\right)}\right\rangle\left|\phi_{a}\right\rangle\left\langle\psi_{j_{n}}^{(n)}\right|\left\langle\phi_{b}\right|,
\end{aligned}
$$

where $n_{ \pm} \equiv\left\{n_{\alpha} \pm \delta_{\alpha \gamma}\right\}$ and $E_{n_{ \pm}}$are the energies of the hardmode states $\left|\left\{n_{\alpha} \pm \delta_{\alpha \gamma}\right\}\right\rangle$ as measured in the asymptotic region. These operators can play the role of annihilation and creation operators in the space spanned by the coarse-grained states. In particular, their matrix elements are

$$
\begin{gathered}
\left\langle\left\langle\left\{\kappa_{\alpha}\right\}\left\|\tilde{b}_{\gamma}\right\|\left\{\lambda_{\alpha}\right\}\right\rangle=\sqrt{\lambda_{\gamma}} \delta_{\left\{\kappa_{\alpha}\right\}\left\{\lambda_{\alpha}-\delta_{\alpha \gamma}\right\}},\right. \\
\left\langle\left\langle\left\{\kappa_{\alpha}\right\}\left\|\tilde{b}_{\gamma}^{\dagger}\right\|\left\{\lambda_{\alpha}\right\}\right\rangle\right\rangle=\sqrt{\lambda_{\gamma}+1} \delta_{\left\{\kappa_{\alpha}\right\}\left\{\lambda_{\alpha}+\delta_{\alpha \gamma}\right\}},
\end{gathered}
$$

up to corrections of order $1 / e^{\# M^{2} l_{\mathrm{P}}^{2}}$. It is important to notice, however, that these operators do not satisfy the exact algebra 
of annihilation and creation operators at the microscopic level. Indeed,

$$
\begin{aligned}
{\left[\tilde{b}_{\beta}, \tilde{b}_{\gamma}^{\dagger}\right]=} & \delta_{\beta \gamma}\left(\sum_{m} e^{-E_{m} / T_{\mathrm{H}}}\right) \sum_{n} e^{E_{n} / T_{\mathrm{H}}} \sum_{i_{n}=1}^{e^{S_{\mathrm{bh}}\left(M-E_{n}\right)}} \sum_{j_{n}=1}^{e_{\mathrm{bh}}\left(M-E_{n}\right)} \\
& \times \sum_{a=1}^{e^{S_{\mathrm{rad}}}} \sum_{b=1}^{e_{\mathrm{rad}}}\left|\psi_{i_{n}}^{(n)}\right\rangle\left|\phi_{a}\right\rangle\left\langle\psi_{j_{n}}^{(n)}\right|\left\langle\phi_{b}\right|
\end{aligned}
$$

which is not the identity operator for $\beta=\gamma$. It is only in the space of coarse-grained states that these operators obey the algebra of annihilation and creation operators:

$$
\begin{gathered}
\left\langle\left\langle\left\{\kappa_{\alpha}\right\}\left\|\left[\tilde{b}_{\beta}, \tilde{b}_{\gamma}^{\dagger}\right]\right\|\left\{\lambda_{\alpha}\right\}\right\rangle\right\rangle=\delta_{\beta \gamma}, \\
\left\langle\left\langle\left\{\kappa_{\alpha}\right\}\left\|\left[\tilde{b}_{\beta}, \tilde{b}_{\gamma}\right]\right\|\left\{\lambda_{\alpha}\right\}\right\rangle\right\rangle=\left\langle\left\langle\left\{\kappa_{\alpha}\right\}\left\|\left[\tilde{b}_{\beta}^{\dagger}, \tilde{b}_{\gamma}^{\dagger}\right]\right\|\left\{\lambda_{\alpha}\right\}\right\rangle=0,\right.
\end{gathered}
$$

which have corrections only of order $1 / e^{\# M^{2} l_{\mathrm{P}}^{2}}$.

Can other microscopic operators be chosen as the annihilation and creation operators in the effective theory? One might think that any operators mapping a generic microstate of $\left.\left.\|\left\{n_{\alpha}\right\}\right\rangle\right\rangle$ [i.e., a state in Eq. (22) with generic $\left.c_{n i_{n} a}\right]$ to those of $\left.\|\left\{n_{\alpha}-\delta_{\alpha \gamma}\right\}\right\rangle$ and $\left.\left.\|\left\{n_{\alpha}+\delta_{\alpha \gamma}\right\}\right\rangle\right\rangle$ would work as $\tilde{b}_{\gamma}$ and $\tilde{b}_{\gamma}^{\dagger}$, respectively. This is, however, not the case. Since a single coarse-grained state $\left.\left.\|\left\{n_{\alpha}\right\}\right\rangle\right\rangle$ corresponds to many microstates, the state obtained by acting such generic operators to a specific microstate, in particular the state $\left.\left.\|\left\{n_{\alpha}\right\}\right\rangle\right\rangle$ involving the specific coefficients $c_{n i_{n} a}$ appearing in the state of the system in Eq. (5), may not have appropriate inner products with the corresponding states $\left.\left.\|\left\{m_{\alpha}\right\}\right\rangle\right\rangle$ 's $\left(\left\{m_{\alpha}\right\} \neq\left\{n_{\alpha}\right\}\right)$ involving the same $c_{n i_{n} a}$. This would mean that those microscopic operators do not serve as annihilation and creation operators in the effective theory erected on the state having these specific coefficients $c_{n i_{n} a}$.

As an example, consider the set of candidate operators

$$
\begin{aligned}
& \tilde{b}_{\gamma} \stackrel{?}{=} c \sum_{n} \sqrt{n_{\gamma}}\left|f^{\left(n_{-}\right)}\right\rangle\left\langle g^{(n)}\right|, \\
& \tilde{b}_{\gamma}^{\dagger} \stackrel{?}{=} c^{*} \sum_{n} \sqrt{n_{\gamma}+1}\left|g^{\left(n_{+}\right)}\right\rangle\left\langle f^{(n)}\right|,
\end{aligned}
$$

where $c$ is a normalization constant, and

$$
\left|f^{(n)}\right\rangle=\sum_{i_{n}=1}^{e^{S_{\mathrm{bh}}\left(M-E_{n}\right)}} f_{n i_{n}}\left|\psi_{i_{n}}^{(n)}\right\rangle, \quad\left|g^{(n)}\right\rangle=\sum_{i_{n}=1}^{e^{S_{\mathrm{bh}}\left(M-E_{n}\right)}} g_{n i_{n}}\left|\psi_{i_{n}}^{(n)}\right\rangle
$$

with generic coefficients satisfying $\quad \sum_{i_{n}}\left|f_{n i_{n}}\right|^{2}=$ $\sum_{i_{n}}\left|g_{n i_{n}}\right|^{2}=1$. This gives

$$
\begin{aligned}
&\left\langle\left\langle\left\{\kappa_{\alpha}\right\}\left\|\tilde{b}_{\gamma}\right\|\left\{\lambda_{\alpha}\right\}\right\rangle=\right. c \sqrt{\lambda_{\gamma}} \delta_{\left\{\kappa_{\alpha}\right\}\left\{\lambda_{\alpha}-\delta_{\alpha \gamma}\right\}} \\
& \times O\left(\frac{1}{\sqrt{e^{S_{\mathrm{bh}}\left(M-E_{\kappa}\right)} e^{S_{\mathrm{bh}}\left(M-E_{\lambda}\right)} e^{S_{\mathrm{rad}}}}}\right), \\
&\left\langle\left\langle\left\{\kappa_{\alpha}\right\}\left\|\tilde{b}_{\gamma} \tilde{b}_{\gamma}^{\dagger}\right\|\left\{\lambda_{\alpha}\right\}\right\rangle\right\rangle=|c|^{2}\left(\lambda_{\gamma}+1\right) \delta_{\left\{\kappa_{\alpha}\right\}\left\{\lambda_{\alpha}\right\}} O\left(\frac{1}{e^{S_{\mathrm{bh}}\left(M-E_{\lambda}\right)}}\right),
\end{aligned}
$$

and there is no choice of $c$ that can make both of these relations compatible with the algebra in the effective theory.

The consideration above provides an argument for the necessity of the dependence of the microscopic operators $\tilde{b}_{\gamma}$ and $\tilde{b}_{\gamma}^{\dagger}$ on the state of the system, in particular $c_{n i_{n} a}$ in Eq. (5). This, however, still allows for operators other than those in Eqs. (29) and (30).

Let us consider the operators

$$
\begin{aligned}
\tilde{b}_{\gamma}= & c \sum_{n} \sqrt{n_{\gamma}} e^{\left(E_{n_{-}}+E_{n}\right) / 2 T_{\mathrm{H}}} \sum_{i_{n_{-}}=1}^{e^{S_{\mathrm{hb}}\left(M-E_{n_{-}}\right)}} \sum_{j_{n}=1}^{S_{\mathrm{bh}}\left(M-E_{n}\right)} \\
& \times \sum_{a=1}^{e^{S_{\mathrm{rad}}}} c_{n_{-} i_{n_{-}} a} c_{n j_{n} a}^{*}\left|\psi \psi_{n_{n_{-}}}^{\left(n_{-}\right)}\right\rangle\left\langle\psi \psi_{j_{n}}^{(n)}\right|, \\
\tilde{b}_{\gamma}^{\dagger}= & c \sum_{n} \sqrt{n_{\gamma}+1} e^{\left(E_{n_{+}}+E_{n}\right) / 2 T_{\mathrm{H}}} \sum_{i_{n_{+}}=1}^{e_{\mathrm{Shh}}^{\left(M-E_{\left.n_{+}\right)}\right)}} \sum_{j_{n}=1}^{S_{\mathrm{bh}\left(M-E_{n}\right)}} \\
& \times \sum_{a=1}^{e^{S_{\mathrm{rad}}} c_{n_{+} i_{n_{+}} a} c_{n j_{n} a}^{*}\left|\psi_{i_{n_{+}}}^{\left(n_{+}\right)}\right\rangle\left\langle\psi_{j_{n}}^{(n)}\right|,}
\end{aligned}
$$

where $c$ is a real number. Note that the combinations of

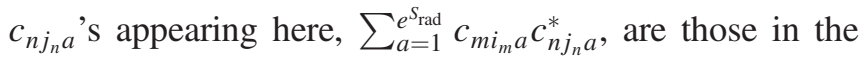
reduced density matrix for the hard and soft modes

$$
\begin{aligned}
& \operatorname{Tr}_{\mathrm{rad}}|\Psi(M)\rangle\langle\Psi(M)| \\
& =\sum_{m} \sum_{n} \sum_{i_{m}=1}^{e^{S_{\mathrm{bh}}\left(M-E_{m}\right)}} \sum_{j_{n}=1}^{S_{\mathrm{bh}}^{\left(M-E_{n}\right)}} \\
& \quad \times \sum_{a=1}^{e_{\mathrm{rad}}} c_{m i_{m} a} c_{n j_{n} a}^{*}\left|\left\{m_{\alpha}\right\}\right\rangle\left|\psi_{i_{m}}^{(m)}\right\rangle\left\langle\left\{n_{\alpha}\right\}\right|\left\langle\psi_{j_{n}}^{(n)}\right|,
\end{aligned}
$$

so that they can be determined purely from the state in the black hole region.

With this choice of $\tilde{b}_{\gamma}$ and $\tilde{b}_{\gamma}^{\dagger}$, we obtain

$$
\left\langle\left\{\left\{\kappa_{\alpha}\right\}\left\|\tilde{b}_{\gamma}\right\|\left\{\lambda_{\alpha}\right\}\right\rangle\right\rangle=\frac{c}{e^{S_{\mathrm{rad}}} \sum_{m} e^{-E_{m} / T_{\mathrm{H}}}} \sqrt{\lambda_{\gamma}} \delta_{\left\{\kappa_{\alpha}\right\}\left\{\lambda_{\alpha}-\delta_{\alpha \gamma}\right\}},
$$




$$
\left\langle\left\langle\left\{\kappa_{\alpha}\right\}\left\|\tilde{b}_{\gamma}^{\dagger}\right\|\left\{\lambda_{\alpha}\right\}\right\rangle\right\rangle=\frac{c}{e^{S_{\mathrm{rad}}} \sum_{m} e^{-E_{m} / T_{\mathrm{H}}}} \sqrt{\lambda_{\gamma}+1} \delta_{\left\{\kappa_{\alpha}\right\}\left\{\lambda_{\alpha}+\delta_{\alpha \gamma}\right\}},
$$

and

$$
\begin{aligned}
\left\langle\left\langle\left\{\kappa_{\alpha}\right\}\left\|\tilde{b}_{\gamma} \tilde{b}_{\gamma}^{\dagger}\right\|\left\{\lambda_{\alpha}\right\}\right\rangle=\right. & \left(\frac{c}{e^{S_{\mathrm{rad}}} \sum_{m} e^{-E_{m} / T_{\mathrm{H}}}}\right)^{2}\left(\lambda_{\gamma}+1\right) \delta_{\left\{\kappa_{\alpha}\right\}\left\{\lambda_{\alpha}\right\}} \\
& \times\left[1+O\left(\frac{e^{S_{\mathrm{rad}}}}{e^{S_{\mathrm{bh}}\left(M-E_{\lambda}\right)}}\right)\right], \\
\left\langle\left\langle\left\{\kappa_{\alpha}\right\}\left\|\tilde{b}_{\gamma}^{\dagger} \tilde{b}_{\gamma}\right\|\left\{\lambda_{\alpha}\right\}\right\rangle=\right. & \left(\frac{c}{e^{S_{\mathrm{rad}}} \sum_{m} e^{-E_{m} / T_{\mathrm{H}}}}\right)^{2} \lambda_{\gamma} \delta_{\left\{\kappa_{\alpha}\right\}\left\{\lambda_{\alpha}\right\}} \\
& \times\left[1+O\left(\frac{e^{S_{\mathrm{rad}}}}{e^{S_{\mathrm{bh}}\left(M-E_{\lambda}\right)}}\right)\right],
\end{aligned}
$$

up to corrections of order $1 / e^{\# M^{2} l_{\mathrm{P}}^{2}}$. We thus find that for

$$
e^{S_{\mathrm{rad}}} \ll e^{S_{\mathrm{bh}}\left(M-E_{\lambda}\right)} \approx e^{S_{\mathrm{bh}}(M)},
$$

the second terms in the square brackets in Eqs. (45) and (46) are negligible, so that the $\tilde{b}_{\gamma}$ and $\tilde{b}_{\gamma}^{\dagger}$ with

$$
c=e^{S_{\mathrm{rad}}} \sum_{m} e^{-E_{m} / T_{\mathrm{H}}}
$$

can play the role of the annihilation and creation operators in the effective theory. In fact, we can show that these operators also satisfy the required commutation relations in Eqs. (34) and (35) when the condition in Eq. (47) is satisfied, namely when the black hole is young.

On the other hand, if the black hole is old, i.e., $e^{S_{\text {rad }}} \gg e^{S_{\text {bh }}(M)}$, then the second terms in the square brackets dominate in Eqs. (45) and (46), jeopardizing the possibility for the $\tilde{b}_{\gamma}$ and $\tilde{b}_{\gamma}^{\dagger}$ to serve as the annihilation and creation operators in the effective theory for any choice of $c$. Technically, this is because the factor obtained by acting $b_{\gamma}^{\dagger}$ or $b_{\gamma}$ to $\left.\left.\|\left\{\lambda_{\alpha}\right\}\right\rangle\right\rangle$

$$
\sum_{i_{\lambda_{ \pm}}=1}^{e^{S_{\mathrm{bh}}\left(M-E_{\lambda_{ \pm}}\right)}} \sum_{j_{\lambda}=1}^{e^{S_{\mathrm{bh}}\left(M-E_{\lambda}\right)}} \sum_{a=1}^{e^{S_{\text {rad }}}} \sum_{b=1}^{e^{S_{\text {rad }}}} c_{\lambda_{ \pm} i_{\lambda_{ \pm}} a} c_{\lambda j_{\lambda} a}^{*} c_{\lambda j_{\lambda} b}\left|\psi_{i_{\lambda_{ \pm}}}^{\left(\lambda_{ \pm}\right)}\right\rangle\left|\phi_{b}\right\rangle
$$

is dominated by the $a=b$ terms if and only if the condition in Eq. (47) is met, giving the state proportional to

$$
\sum_{i_{\lambda_{ \pm}}=1}^{e^{S_{\mathrm{bh}}\left(M-E_{\lambda_{ \pm}}\right)}} \sum_{a=1}^{e^{S_{\mathrm{rad}}}} c_{\lambda_{ \pm} i_{\lambda_{ \pm}} a}\left|\psi_{i_{\lambda_{ \pm}}}^{\left(\lambda_{ \pm}\right)}\right\rangle\left|\phi_{a}\right\rangle
$$

and hence to the $\left.\left.\|\left\{\lambda_{\alpha} \pm \delta_{\alpha \gamma}\right\}\right\rangle\right\rangle$ obtained using the specific $c_{n j_{n}} a$ 's appearing in the state of the system. This shows how the Page time can be relevant in the construction of the interior operators, despite the fact that the hard-mode and radiation states take a separable form as in Eq. (13) throughout the history of the black hole. ${ }^{3}$ In fact, for an old black hole, we do not see how one can construct the appropriate annihilation and creation operators in the effective theory using only the information in the black hole reduced density matrix in Eq. (42).

Incidentally, a construction of $\tilde{b}_{\gamma}$ and $\tilde{b}_{\gamma}^{\dagger}$ involving only radiation states is not possible. What allowed the construction of operators in Eqs. (40) and (41) is the correlations between the attributes of the hard and soft modes coming from the constraints imposed on the black hole vacuum state (the requirement that it does not have any features associated with semiclassical excitations). Such correlations do not exist between the hard modes and radiation.

As discussed in Ref. [8], the effective theory of the interior erected as above describes only a limited spacetime region: the causal domain of the union of the zone and its mirror region on the spatial hypersurface at $t$ (the time at which the effective theory is erected) in the effective twosided geometry. The black hole singularity may be regarded as a manifestation of the fact that this theory is obtained by coarse graining and hence represents a finite-dimensional, nonunitary system. Specific operators used in Eqs. (26) and (27), for example those in Eqs. (29) and (30), are selected presumably because they correspond to observables which classicalize within such a finite-dimensional system [9]. Locality seems to play a key role in this quantum-toclassical transition.

The fact that an effective theory represents only a limited spacetime region implies that the picture of the whole interior, as described by general relativity, can be obtained only by using multiple effective theories erected at different times. This is the sense in which the global spacetime in general relativity emerges from the microscopic description.

\section{A. Relation to the work by Papadodimas and Raju}

A construction of interior operators similar to the one described here, based on the doubled Hilbert space structure, was considered in the well-known work by Papadodimas and Raju [23-25]. While the mathematical structures of the two are related (at the level of dividing the system into two components), their physical implementations are different in several key aspects, leading to different solutions to the firewall paradoxes $[7,12,13]$.

In Ref. [23], the doubled Hilbert space structure was obtained by coarse graining bulk fields (generalized free fields in CFT) to separate the degrees of freedom represented by one of the Hilbert space factors, which

\footnotetext{
${ }^{3}$ This separation is not a statistical statement; i.e., it does not receive exponentially small corrections from statistics. A similar statement applies for a state of the form in Eq. (62) in the next section.
} 
increase as the black hole evaporates. [Note that this coarse graining is different from that discussed in Eqs. (20)-(22).] In contrast, our hard modes are selected from the black hole degrees of freedom by an energetic criterion, which thus decrease as the evaporation progresses. These modes have gaps larger than the Hawking temperature (instead of the effectively continuous spectrum envisioned in Ref. [23]), which plays an important role in the picture as we have seen in this and the previous sections. This leads, for example, to an entanglement structure different from that envisioned in the earlier work; in particular, the hard-mode and radiation states take a separable form throughout the history of the black hole.

In Refs. [24,25], which are supposed to subsume the earlier construction in Ref. [23], the "state dependence" of the map between boundary operators and bulk local operators was invoked to address the firewall paradoxes. Problems of the state dependence- the frozen vacuum and Born rule problems-were discussed in Refs. [14] and [15], respectively. In our construction, interior operators do depend on the microstate on which they are built, in the sense that operators $\tilde{b}_{\gamma}$ and $\tilde{b}_{\gamma}^{\dagger}$ depend on $c_{n i_{n} a}$ as shown in Eqs. (29) and (30) and Eqs. (40) and (41). As discussed in Ref. [9], however, the fact that our hard modes are selected energetically (which is also the case in a large AdS black hole as discussed in the next section) allows us to avoid the problems in Refs. [14,15].

Specifically, consider the space $\mathcal{H}_{M}$ of pure states in which the energy $E$ in a spatial region is bounded by $E<M$, where $M$ is sufficiently large that a typical state in $\mathcal{H}_{M}$ is a black hole state. We then consider the space of all states that are obtained by acting appropriately smoothed hard-mode (semiclassical) operators on any of the black hole microstates in $\mathcal{H}_{M}$ and have energies smaller than $M+\delta E$. This space, denoted by $B_{\delta E} \mathcal{H}_{M}$, has dimension $e^{S_{\mathrm{bh}}(M)+S_{\mathrm{exc}}}$, where $S_{\mathrm{exc}}$ is the entropy of the possible semiclassical excitations. One can then show that a typical state $|\psi\rangle$ in $\mathcal{H}_{M+\delta E}$ can be written as

$$
|\psi\rangle=\sin \theta\left|\psi_{\mathrm{exc}}\right\rangle+\cos \theta\left|\psi_{\mathrm{vac}}\right\rangle
$$

with

$$
\sin ^{2} \theta \sim e^{-\left(\delta E / T_{\mathrm{H}}-S_{\mathrm{exc}}\right)} .
$$

Here, $\left|\psi_{\text {exc }}\right\rangle$ and $\left|\psi_{\text {vac }}\right\rangle$ are elements of $B_{\delta E} \mathcal{H}_{M}$ and its complement $\mathcal{H}_{M+\delta E} / B_{\delta E} \mathcal{H}_{M}$, respectively, and $T_{\mathrm{H}}=$ $1 / 8 \pi M l_{\mathrm{p}}^{2}$. Assuming that semiclassical excitations are well within the Bekenstein bound [26,27], i.e., $S_{\mathrm{exc}}<\delta E / T_{\mathrm{H}}$ with $\left(\delta E / T_{\mathrm{H}}-S_{\mathrm{exc}}\right) / S_{\mathrm{exc}} \ll 1,{ }^{4}$ and that a semiclassical excitation has entropy of order a few or larger, we obtain

\footnotetext{
${ }^{4}$ This assumption was also used implicitly in obtaining Eq. (8).
}

$$
\text { a few } \lesssim S_{\text {exc }}<\frac{\delta E}{T_{\mathrm{H}}} \quad \Rightarrow \quad \sin ^{2} \theta \ll 1
$$

We thus find, unlike the claim in Ref. [15], that a state having excitations over a semiclassical black hole background is atypical in the microscopic Hilbert space.

The direct application of the above analysis is limited to the excitations outside the horizon, i.e., the states obtained by acting $b_{\gamma}^{\dagger}$ 's, which raise the energy of the state. On the other hand, an object in the interior, excited by $a_{\xi}^{\dagger}$, s, involves operators $\tilde{b}_{\gamma}^{\dagger}$, which lower the energy as measured in the asymptotic region. This is reflected in the fact that the infalling Hamiltonian $H$ in Eq. (28) does not commute with the generator of time evolution in the distant description, so that a positive energy excitation in the zone will develop negative energy components "after passing the horizon." However, the conclusion that a semiclassically excited state is atypical still persists if we focus only on excitations thrown from the exterior, i.e., the states that can be obtained by acting the infalling time evolution operator $U=e^{-i H \tau}$ on the states considered in the previous paragraph, ${ }^{5}$ since the operator $U$ is approximately unitary over the relevant timescale $\tau$.

This implies that the Hilbert space for semiclassical excitations, $\mathcal{H}_{\text {exc }}$, built on each of the orthogonal black hole and radiation microstates need not overlap significantly with each other. This is indeed expected to be the case from genericity consideration; we can show that states representing the same semiclassical excitation but built on different orthogonal microstates $A$ and $B$ have overlap

$$
{ }_{A}\left\langle\Psi(M)\left|\mathcal{O}_{\delta E}^{(A) \dagger} \mathcal{O}_{\delta E}^{(B)}\right| \Psi(M)\right\rangle_{B} \approx O\left(\frac{1}{\sqrt{e^{S_{\mathrm{bh}}(M)} e^{S_{\mathrm{rad}}}}} e^{-\delta E / 2 T_{\mathrm{H}}}\right),
$$

where $\mathcal{O}_{\delta E}^{(A, B)}$ are the operators that excite an appropriately smoothed semiclassical mode of energy $\delta E$ (either in a distant or infalling frame). We find that this level of suppression is sufficient for us to be able to treat the microscopic Hilbert space as

$$
\mathcal{H} \approx \mathcal{H}_{\text {exc }} \otimes \mathcal{H}_{\text {vac }}
$$

where the elements of $\mathcal{H}_{\mathrm{vac}}$ cannot be discriminated as quantum degrees of freedom in the semiclassical theory. Note that this structure is different from that considered in Refs. [14,15,23-25]. In particular, we can define global

\footnotetext{
${ }^{5}$ This excludes certain configurations that are obtained by sending signals from the second exterior in a genuinely two-sided black hole. In the context of a collapse-formed black hole, such configurations correspond to the states obtained by performing highly fine-tuned and complicated operations to the soft modes and/or radiation.
} 
operators $\mathcal{O}=b_{\gamma}, b_{\gamma}^{\dagger}, a_{\xi}, a_{\xi}^{\dagger}$ that act linearly throughout the space of all semiclassical states built on each of the vacuum microstates $^{6}$

$$
\mathcal{O}=\sum_{A=1}^{e^{S_{\mathrm{bh}}(M)+S_{\mathrm{rad}}}} \sum_{I} \sum_{J}\left|\Psi_{I}^{(A)}\right\rangle \mathcal{O}_{I J}^{(A)}\left\langle\Psi_{J}^{(A)}\right|
$$

where $I$ and $J$ are the indices specifying semiclassical states (regardless of the microstate), $A$ runs over orthogonal vacuum microstates of the form in Eq. (5), $\left|\Psi_{I}^{(A)}\right\rangle$ is the semiclassical state $I$ built on microstate $A$, and $\mathcal{O}_{I J}^{(A)}$ is the matrix element of the corresponding operator built on $A-b_{\gamma}^{(A)}, b_{\gamma}^{(A) \dagger}, a_{\xi}^{(A)}, a_{\xi}^{(A) \dagger}$-in the microscopic Hilbert space. These global operators $\mathcal{O}$ obey the correct annihilation and creation operator algebra up to corrections exponentially suppressed in $\delta E / T_{\mathrm{H}}$, which is typically huge for a small object falling into the black hole.

Finally, we have divided the system into three components - the hard modes, soft modes, and radiation - rather than two. This allows us to impose an energy constraint in the black hole system, which is essential in deriving the properties of operators in the effective theory described above. It also elucidates a difference between our construction and that of Refs. [23-25]; our effective second-exterior operators $\tilde{b}_{\gamma}$ and $\tilde{b}_{\gamma}^{\dagger}$ are constructed as the mirror of hard modes, while those in Refs. [23-25] as the mirror of a system including early radiation. This makes, for example, the vanishing of commutators between $\tilde{b}_{\gamma} / \tilde{b}_{\gamma}^{\dagger}$ and operators acting on early radiation not automatic.

We may, however, expect that any simple operation performed on early radiation does not affect the black hole interior, protecting locality at the semiclassical level. This possibility has recently been studied in Ref. [28] using the concept of computational complexity. These authors have analyzed the problem for a state without an energy constraint [a state of the form in Eq. (62) in the next section]; here we discuss the issue using a state, Eq. (5), that has a physically relevant form in our framework. Consider the microscopic-level expression [see Eq. (22)] of a coarsegrained state $\left.\left.\|\left\{n_{\alpha}\right\}\right\rangle\right\rangle$ built on microstate $A$

$$
\begin{aligned}
\left.\left.\|\left\{n_{\alpha}\right\}\right\rangle\right\rangle_{A}= & e^{E_{n} / 2 T_{\mathrm{H}}} \sqrt{\sum_{m} e^{-E_{m} / T_{\mathrm{H}}}} \sum_{i_{n}=1}^{S_{\mathrm{bh}}\left(M-E_{n}\right)} \\
& \times \sum_{a=1}^{e^{S_{\mathrm{rad}}}} c_{n i_{n} a}^{(A)}\left|\psi i_{i_{n}}^{(n)}\right\rangle\left|\phi_{a}\right\rangle .
\end{aligned}
$$

\footnotetext{
${ }^{6}$ These operators can be easily extended to linear operators throughout the whole microscopic Hilbert space. It is simply that they then cannot be interpreted as annihilation and creation operators outside the space of semiclassical states built on each vacuum microstate.
}

A general operation on early radiation can be expressed as a quantum channel

$$
\left.\mathcal{E}\left[\|\left\{n_{\alpha}\right\}\right\rangle_{A A}\left\langle\left\langle\left\{n_{\alpha}\right\} \|\right]=\sum_{x} E_{x} \|\left\{n_{\alpha}\right\}\right\rangle\right\rangle_{A A}\left\langle\left\{n_{\alpha}\right\} \| E_{x}^{\dagger},\right.
$$

where $E_{x}$ are Kraus operators that act on radiation as

$$
E_{x}=\sum_{a=1}^{e^{S_{\mathrm{rad}}}} \sum_{b=1}^{e_{\mathrm{rad}}}\left|\phi_{a}\right\rangle E_{a b}^{x}\left\langle\phi_{b}\right|,
$$

which satisfy $\sum_{x} E_{x}^{\dagger} E_{x}=\mathbb{I}$.

Let us now consider the matrix element of this state between coarse-grained states $\left.\|\left\{\kappa_{\alpha}\right\}\right\rangle$ and $\left.\left.\|\left\{\lambda_{\alpha}\right\}\right\rangle\right\rangle$ built on microstate $B$ and $C$, respectively. This gives

$$
\begin{aligned}
{ }_{B}\left\langle\left\langle\left\{\kappa_{\alpha}\right\} \| \mathcal{E}\left[\|\left\{n_{\alpha}\right\}\right\rangle_{A_{A}}\left\langle\left\langle\left\{n_{\alpha}\right\} \|\right] \|\left\{\lambda_{\alpha}\right\}\right\rangle_{C}\right.\right. \\
=e^{2 E_{n} / T_{\mathrm{H}}}\left(\sum_{m} e^{-E_{m} / T_{\mathrm{H}}}\right)^{2} \delta_{\left\{\kappa_{\alpha}\right\}\left\{n_{\alpha}\right\}} \delta_{\left\{\lambda_{\alpha}\right\}\left\{n_{\alpha}\right\}} \\
\times \sum_{x}\left[\sum_{i_{n}=1}^{e^{S_{\mathrm{bh}}\left(M-E_{n}\right)}} \sum_{a=1}^{e_{\text {rad }}^{S_{\text {rad }}}} \sum_{b=1}^{e_{\text {rad }}} c_{n i_{n} a}^{(B) *} E_{a b}^{x} c_{n i_{n} b}^{(A)}\right] \\
\times\left[\sum_{j_{n}=1}^{e^{S_{\mathrm{hh}}\left(M-E_{n}\right)}} \sum_{c=1}^{e_{\text {rad }}^{S_{\text {rad }}}} \sum_{d=1}^{S_{\text {rad }}} c_{n j_{n} c}^{(A) *} E_{c d}^{x} c_{n j_{n} d}^{(C)}\right] .
\end{aligned}
$$

We thus find that the condition for the coarse-grained states at the semiclassical level—and hence the interior- not to be affected by any measurement made by a remote observer is

$$
\begin{aligned}
& e^{E_{n} / T_{\mathrm{H}}}\left(\sum_{m} e^{-E_{m} / T_{\mathrm{H}}}\right) \sum_{i_{n}=1}^{e^{S_{\mathrm{bh}}\left(M-E_{n}\right)}} \sum_{a=1}^{S_{\mathrm{rad}}} \sum_{b=1}^{e_{\mathrm{rad}}} c_{n i_{n} a}^{(A) *} E_{a b}^{x} c_{n i_{n} b}^{(B)} \\
& =q_{x} U_{A B}^{x}
\end{aligned}
$$

for all $n=\left\{n_{\alpha}\right\}, A, B$, and $x$, up to exponentially suppressed corrections. Here, $U_{A B}^{x}$ are the elements of (arbitrary) unitary matrices $U^{x}$ acting on the space of microstates, and $q_{x}$ are numbers satisfying $\sum_{x}\left|q_{x}\right|^{2}=1$. This is morally the condition for the simplicity of the measurement and pseudorandomness of Hawking radiation discussed in Ref. [28], which we expect to be satisfied under assumptions similar to those adopted there. Assuming this is true, our framework preserves locality at the semiclassical level; i.e., any observer performing a simple operation on early radiation cannot remotely affect the black hole interior. ${ }^{7}$

\footnotetext{
${ }^{7}$ If an observer performs an operation with superpolynomial complexity, he or she can affect the state of the black hole at the semiclassical level; in fact, they can even create a firewall state. Even in this case, however, the black hole quickly "repairs" itself and recover the smooth horizon in a timescale of order the scrambling time $[8,9]$.
} 


\section{LARGE ADS BLACK HOLE}

After the original submission of this paper, ${ }^{8}$ an interesting paper by Penington, Shenker, Stanford, and Yang appeared [16] which discusses related issues. In particular, these authors adopted the same machinery as that used here to construct interior operators in Eqs. (40) and (41), which they referred to as the Petz map following the terminology used in quantum information science.

The models employed in Ref. [16] are analogous to a large AdS black hole, which is distinct from a flat space or small AdS black hole. For a large AdS black hole, there are modes whose wavelengths in the angular directions are smaller than the horizon radius and yet which have frequencies smaller than the Hawking temperature, since the inverse Hawking temperature is (much) smaller than the horizon radius, $1 / T_{\mathrm{H}} \ll r_{+}$. Here, $T_{\mathrm{H}}=3 r_{+} / 4 \pi l^{2}$ is the Hawking temperature, and $r_{+}$and $l$ are the horizon and AdS radii, respectively. This is in contrast to the case of a flat space or small AdS black hole, in which $1 / T_{\mathrm{H}}=4 \pi r_{+}$, so a mode having a wavelength in the angular directions smaller than the horizon radius necessarily has a frequency larger than $T_{\mathrm{H}}$.

Let us see what happens if we apply the construction in the previous section to these modes $\left(1 / r_{+} \ll \omega, \Delta \omega \lesssim T_{\mathrm{H}}\right)$, which we call "relatively harder" (but still soft) modes. Since the uncertainty in energy $\left(\sim T_{\mathrm{H}}\right)$ is larger than the frequencies of these modes, their states $\left|\left\{n_{\alpha}\right\}\right\rangle$ need not be correlated with the states $\left|\psi_{i}\right\rangle$ of the other, "relatively softer" (soft) modes $\left(\omega \lesssim 1 / r_{+}\right)$as in Eq. (5). Specifically, the state of the system with the black hole put in the semiclassical vacuum can be written as

$$
|\Psi(M)\rangle=\sum_{n=1}^{e^{S_{\mathrm{h}}}} \sum_{i=1}^{e^{S_{\mathrm{bh}}(M)}} \sum_{a=1}^{e^{S_{\mathrm{rad}}}} c_{\text {nia }}\left|\left\{n_{\alpha}\right\}\right\rangle\left|\psi_{i}\right\rangle\left|\phi_{a}\right\rangle,
$$

where $e^{S_{\mathrm{h}}}\left(\ll e^{S_{\mathrm{bh}}(M)}\right)$ is the dimension of the Hilbert space for the harder modes, $S_{\mathrm{bh}}(M)=\pi\left(2 l^{2} M / l_{\mathrm{P}}\right)^{2 / 3}$ is the density of states for the softer modes $(\approx$ that of the black hole), and $\left|\phi_{a}\right\rangle$ represents states of the auxiliary system to which the AdS system is coupled at the boundary. ${ }^{9}$ Note that $\left|\left\{n_{\alpha}\right\}\right\rangle$ and $\left|\psi_{i}\right\rangle$ here represent the states of the harder and softer soft modes, rather than hard and soft modes. For

\footnotetext{
${ }^{8}$ The original version of this paper was submitted such that it would appear in an earlier announcement (submitted on 26 Nov 2019 14:02:29, EST). The appearance, however, was delayed due to a moderation by arXiv administration, which resulted in a larger arXiv number.

${ }^{9}$ For simplicity, here we have assumed that the energy gaps between different $\left|\phi_{a}\right\rangle$ 's are smaller than of $O\left(T_{\mathrm{H}}\right)$, the precision with which the black hole mass is specified. To see the finiteness of the temperature in the auxiliary system (Hawking radiation), we need to include states of the auxiliary system with energy gaps larger than $T_{\mathrm{H}}$.
}

simplicity, we set all the hard modes to be on their ground states; these modes will be considered later.

We can now define the normalized coarse-grained states along the lines of Eq. (20):

$$
\left.\|\left\{n_{\alpha}\right\}\right\rangle=\sqrt{e^{S_{\mathrm{h}}}} \sum_{i=1}^{e^{S_{\mathrm{bh}}(M)}} \sum_{a=1}^{e^{S_{\mathrm{rad}}}} c_{n i a}\left|\psi_{i}\right\rangle\left|\phi_{a}\right\rangle .
$$

The statistical errors for the normalizations are fractionally of order $1 / \sqrt{e^{S_{\mathrm{bh}}(M)} e^{S_{\mathrm{rad}}}} \sim 1 / e^{S_{\mathrm{sys}}}$; here and below, we denote contributions of order $1 / e^{\# S_{\text {bh }}+\# S_{\text {rad }}}$ simply by $1 / e^{S_{\mathrm{sys}}}$. We then find that the softer-mode states that are entangled with different harder-mode states have nonzero overlaps

$$
\left\langle\left\{\kappa_{\alpha}\right\} \|\left\{\lambda_{\alpha}\right\}\right\rangle=O\left(\frac{1}{\sqrt{e^{S_{\mathrm{bh}}} e^{S_{\mathrm{rad}}}}}\right) \quad \text { for }\left\{\kappa_{\alpha}\right\} \neq\left\{\lambda_{\alpha}\right\},
$$

although they are small, of order $1 / e^{S_{\mathrm{sys}}}$. This is in contrast to the soft mode states entangled with the hard-mode states in Secs. II and III, whose overlaps were virtually zero as in Eq. (9).

These small overlaps allow us, after the Page time, to choose " $\tilde{b}_{\gamma}$ and $\tilde{b}_{\gamma}^{\dagger}$ operators" for the harder soft modes which act only on the auxiliary system, i.e., early radiation. Consider operators

$$
\begin{aligned}
& \tilde{b}_{\gamma}=e^{S_{\mathrm{h}}+S_{\mathrm{bh}}} \sum_{n=1}^{e^{S_{\mathrm{h}}}} \sqrt{n_{\gamma}} \sum_{i=1}^{e^{S_{\mathrm{bh}}}} \sum_{a=1}^{e^{S_{\text {rad }}}} \sum_{b=1}^{e_{\text {rad }}} c_{n_{-} i a} c_{n i b}^{*}\left|\phi_{a}\right\rangle\left\langle\phi_{b}\right|, \\
& \tilde{b}_{\gamma}^{\dagger}=e^{S_{\mathrm{h}}+S_{\mathrm{bh}}} \sum_{n=1}^{e^{S_{\mathrm{h}}}} \sqrt{n_{\gamma}+1} \sum_{i=1}^{e^{S_{\mathrm{bh}}}} \sum_{a=1}^{e_{\text {rad }}} \sum_{b=1}^{S_{\mathrm{rad}}} c_{n_{+} i a} c_{n i b}^{*}\left|\phi_{a}\right\rangle\left\langle\phi_{b}\right| .
\end{aligned}
$$

This leads to

$$
\begin{gathered}
\left\langle\left\langle\left\{\kappa_{\alpha}\right\}\left\|\tilde{b}_{\gamma}\right\|\left\{\lambda_{\alpha}\right\}\right\rangle\right\rangle=\sqrt{\lambda_{\gamma}} \delta_{\left\{\kappa_{\alpha}\right\}\left\{\lambda_{\alpha}-\delta_{\alpha \gamma}\right\}}, \\
\left\langle\left\langle\left\{\kappa_{\alpha}\right\}\left\|\tilde{b}_{\gamma}^{\dagger}\right\|\left\{\lambda_{\alpha}\right\}\right\rangle\right\rangle=\sqrt{\lambda_{\gamma}+1} \delta_{\left\{\kappa_{\alpha}\right\}\left\{\lambda_{\alpha}+\delta_{\alpha \gamma}\right\}},
\end{gathered}
$$

and

$$
\begin{aligned}
\left\langle\left\langle\left\{\kappa_{\alpha}\right\}\left\|\tilde{b}_{\beta} \tilde{b}_{\gamma}^{\dagger}\right\|\left\{\lambda_{\alpha}\right\}\right\rangle=\right. & \sqrt{\lambda_{\beta}+\delta_{\beta \gamma}} \sqrt{\lambda_{\gamma}+1} \delta_{\left\{\kappa_{\alpha}\right\}\left\{\lambda_{\alpha}-\delta_{\alpha \beta}+\delta_{\alpha \gamma}\right\}} \\
& \times\left[1+O\left(\frac{e^{S_{\mathrm{bh}}}}{e^{S_{\mathrm{rad}}}}\right) \delta_{\left\{\kappa_{\alpha}\right\}\left\{\lambda_{\alpha}\right\}}\right], \\
\left\langle\left\langle\left\{\kappa_{\alpha}\right\}\left\|\tilde{b}_{\beta}^{\dagger} \tilde{b}_{\gamma}\right\|\left\{\lambda_{\alpha}\right\}\right\rangle=\right. & \sqrt{\lambda_{\beta}-\delta_{\beta \gamma}+1} \sqrt{\lambda_{\gamma}} \delta_{\left\{\kappa_{\alpha}\right\}\left\{\lambda_{\alpha}+\delta_{\alpha \beta}-\delta_{\alpha \gamma}\right\}} \\
& \times\left[1+O\left(\frac{e^{S_{\mathrm{bh}}}}{e^{S_{\mathrm{rad}}}}\right) \delta_{\left\{\kappa_{\alpha}\right\}\left\{\lambda_{\alpha}\right\}}\right]
\end{aligned}
$$




$$
\left.\left\langle\left\{\kappa_{\alpha}\right\}\left\|\tilde{b}_{\beta} \tilde{b}_{\gamma}\right\|\left\{\lambda_{\alpha}\right\}\right\rangle\right\rangle=\sqrt{\lambda_{\beta}-\delta_{\beta \gamma}} \sqrt{\lambda_{\gamma}} \delta_{\left\{\kappa_{\alpha}\right\}\left\{\lambda_{\alpha}-\delta_{\alpha \beta}-\delta_{\alpha \gamma}\right\}},
$$

$$
\begin{aligned}
\left\langle\left\{\kappa_{\alpha}\right\}\left\|\tilde{b}_{\beta}^{\dagger} \tilde{b}_{\gamma}^{\dagger}\right\|\left\{\lambda_{\alpha}\right\}\right\rangle= & \sqrt{\lambda_{\beta}+\delta_{\beta \gamma}+1} \\
& \times \sqrt{\lambda_{\gamma}+1} \delta_{\left\{\kappa_{\alpha}\right\}\left\{\lambda_{\alpha}+\delta_{\alpha \beta}+\delta_{\alpha \gamma}\right\}},
\end{aligned}
$$

where we have omitted contributions suppressed by $1 / e^{S_{\mathrm{sys}}}$. In order for the operators in Eqs. (65) and (66) to play the role of the annihilation and creation operators, the second terms in the square brackets in Eqs. (69) and (70) must be negligible, which is the case if

$$
e^{S_{\mathrm{bh}}} \ll e^{S_{\mathrm{rad}}},
$$

i.e., the black hole is old. With Eq. (73), the algebra of annihilation and creation operators, Eqs. (34) and (35), is indeed satisfied.

The operators for the harder modes constructed in this way, however, are not relevant in erecting an effective theory of the interior. This is because the majority of the states obtained by "exciting" a given vacuum microstate by these operators correspond simply to other vacuum microstates, i.e., states with different soft-mode configurations. One might think that there are some rare "excitations" generated by acting these operators on some microstate which can meaningfully be considered as being thrown into the black hole. Such excitations, however, are quickly thermalized before hitting the stretched horizon. In fact, they are nothing other than exponentially rare statistical fluctuations of the thermal soft-mode gas, which do not represent a semiclassical object falling into the black hole. This picture is consistent with the analysis of Ref. [29] in which it was shown that excitations having energy $\delta E \ll T_{\mathrm{H}}$ do not significantly affect relevant correlation functions in the bulk.

We therefore end up with the situation similar to the case of a flat space black hole. A semiclassical object falling into the black hole is described by hard modes, which have frequencies $\omega$ and their gaps $\Delta \omega$ larger than $\Delta$ determined by the black hole temperature

$$
\Delta \approx O\left(\frac{r_{+}}{l^{2}}\right)
$$

Assuming that the energy of the black hole system (comprising the hard and soft modes) is specified with maximal precision of order $\Delta$, the state of the entire system is given by Eq. (5), where $\left|\left\{n_{\alpha}\right\}\right\rangle$ and $\left|\psi_{i_{n}}^{(n)}\right\rangle$ represent the states of the hard modes and the corresponding (both harder and softer) soft modes, respectively. We note, however, that the expression for the density of states now takes the form appropriate for a large AdS black hole, $S_{\mathrm{bh}}(M)=$ $\pi\left(2 l^{2} M / l_{\mathrm{P}}\right)^{2 / 3}$.

As in the flat space case, the mass and entropy of the black hole can be viewed as being carried by the soft modes. To see this, we note that the local temperature of these modes in the bulk is given by

$$
T_{\mathrm{loc}}(r)=\frac{T_{\mathrm{H}}}{\sqrt{\frac{r^{2}}{l^{2}}-\frac{r_{+}^{3}}{l^{2} r}}}=\frac{3 r_{+}}{4 \pi l^{2} \sqrt{\frac{r^{2}}{l^{2}}-\frac{r_{+}^{3}}{l^{2} r}}},
$$

and the stretched horizon is located at $r=r_{s}$ with

$$
r_{s}-r_{+} \sim \frac{r_{+} l_{s}^{2}}{l^{2}}
$$

Here, $l_{s}$ is the string length. By integrating their entropy and energy densities, $\sim N T_{\mathrm{loc}}(r)^{3}$ and $\sim N T_{\mathrm{loc}}(r)^{4}$, from the stretched horizon toward the exterior, we find

$$
\begin{gathered}
S \sim N \int_{r_{\mathrm{s}}}^{\infty} T_{\mathrm{loc}}(r)^{3} \frac{r^{2} d r}{\sqrt{\frac{r^{2}}{l^{2}}-\frac{r_{+}^{3}}{l^{2} r}}} \sim \frac{r_{+}^{2}}{l_{\mathrm{P}}^{2}}, \\
E_{\mathrm{loc}} \sim N \int_{r_{s}}^{\infty} T_{\mathrm{loc}}(r)^{4} \frac{r^{2} d r}{\sqrt{\frac{r^{2}}{l^{2}}-\frac{r_{+}^{3}}{l^{2} r}}} \sim \frac{M}{\sqrt{\frac{r_{s}^{2}}{l^{2}}-\frac{r_{+}^{3}}{l^{2} r_{s}}}},
\end{gathered}
$$

where $N$ is the number of low-energy species and we have used the relation $l_{s}^{2} / N \sim l_{\mathrm{P}}^{2}$ and

$$
\frac{l}{\sqrt{r_{+}\left(r_{s}-r_{+}\right)}} \sim \frac{1}{\sqrt{\frac{r_{s}^{2}}{l^{2}}-\frac{r_{+}^{3}}{l^{2} r_{s}}}} .
$$

These indeed reproduce parametrically the entropy of the black hole and the mass $M$ measured at the stretched horizon, $E_{\mathrm{loc}}$, where most of the modes are located. The internal dynamics of the soft modes near the stretched horizon is expected to be nonlocal below the AdS length scale $l$ in the directions along the horizon.

The construction of operators of an effective theory of the interior goes as in Sec. III. Suppose that the state of the system with the black hole put in the semiclassical vacuum is given by Eq. (5) at time $t$. The annihilation and creation operators for the infalling modes, $a_{\xi}$ and $a_{\xi}^{\dagger}$ in Eqs. (26) and (27), can then be constructed by $b_{\gamma}$ and $b_{\gamma}^{\dagger}$ in Eqs. (18) and (19) and $\tilde{b}_{\gamma}$ and $\tilde{b}_{\gamma}^{\dagger}$ in Eqs. (29) and (30). If the black hole is young, i.e., if it is not maximally entangled with the rest of the system, then the operators $\tilde{b}_{\gamma}$ and $\tilde{b}_{\gamma}^{\dagger}$ can be taken to act only on the soft modes as in Eqs. (40) and (41) with Eq. (48). This option, however, is not available if the black hole is old. The erected effective theory describes the physics in the causal domain of the equal-time hypersurface at $t$ in the emergent effective two-sided black hole geometry. 
Finally, we emphasize that the discussion at the end of Sec. III leading to Eq. (55) carries over to the case of a large AdS black hole. Specifically, the overlap $\sin \theta$ between a state in which hard modes are excited by $b_{\gamma}^{\dagger}$ 's and a typical state of the same energy (within uncertainty of order $\left.T_{\mathrm{H}} \sim r_{+} / l^{2}\right)$ is given by

$$
\sin ^{2} \theta \sim e^{-\left(\delta E / T_{\mathrm{H}}-S_{\text {hard }}\right)},
$$

where $\delta E$ and $S_{\text {hard }}$ are the energy and coarse-grained entropy of the hard-mode excitation, respectively. Therefore, assuming that the hard-mode excitation is well within the Bekenstein bound, we obtain

$$
\text { a few } \lesssim S_{\text {hard }}<\frac{\delta E}{T_{\mathrm{H}}} \quad \Rightarrow \quad \sin ^{2} \theta \ll 1 .
$$

Given that the infalling time evolution operator $U=e^{-i H \tau}$ is approximately unitary over the relevant timescale, this translates into the statement that the states which have semiclassical objects falling inside the horizon occupy only a negligible fraction of the microscopic Hilbert space. As discussed in Sec. III, this allows us to treat the microscopic Hilbert space as

$$
\mathcal{H} \approx \mathcal{H}_{\text {exc }} \otimes \mathcal{H}_{\text {vac }},
$$

where $\mathcal{H}_{\text {exc }}$ and $\mathcal{H}_{\text {vac }}$ are the Hilbert space of the effective semiclassical theory and that for the vacuum microstates, respectively. In particular, physically relevant operators in the semiclassical theory- $b_{\gamma}, b_{\gamma}^{\dagger}, a_{\xi}$, and $a_{\xi}^{\dagger}$-can be globally defined as linear operators at the microscopic level, which obey the correct annihilation and creation operator algebra in the space of the semiclassical states up to corrections suppressed by $e^{-\delta E / T_{\mathrm{H}}}$.

\section{A. Relation to entanglement wedge reconstruction and islands}

Recently, there has been significant progress in understanding the interior of a black hole using holographic entanglement wedge reconstruction [17-19]. According to these analyses, operators acting on early radiation are sufficient to reconstruct a portion of the black hole interior after the Page time. On the other hand, we have seen that in order to erect the effective theory of the interior at any given time in a distant description, the interior operators must act on the soft-mode degrees of freedom in addition to the early radiation. How can these two statements be reconciled?

A key to understand this issue is time evolution. In general, entanglement wedge reconstruction assumes that we know the time evolution operator of the holographic theory, i.e., that of a system comprising the boundary theory and any auxiliary theory coupling to it. In addition, the analyses referred to above assume that the information leaked from the boundary theory-representing the bulk with a black hole - to the auxiliary system - a system storing Hawking radiation-is effectively irreversible. These imply that given a state at some time $t$, we can adopt the following strategy to reconstruct the interior. Since a hard mode object that had fallen before some time $t_{\mathrm{w}}(<t)$, as well as the soft modes entangled with it, would be fully mixed into the black hole, and their information is emitted in radiation through time evolution, we can in principle represent (a portion of) the interior spacetime describing the fate of the object on radiation at time $t$. If we have a complete knowledge about the radiation state at $t$, then $t-t_{\mathrm{w}}$ is of the order of the information retention time, but if we lack a part of the knowledge, then $t-t_{w}$ is larger.

This explains why the interior portion of the entanglement wedge of radiation in the work of Refs. [17-19] emerges as an island: the region disconnected from that supporting the radiation. We can indeed check the consistency of this understanding at the level of precision including the coefficients of terms enhanced by the logarithm of the black hole mass or entropy.

For concreteness, let us consider a black hole in fourdimensional asymptotically flat spacetime. Suppose that the state is given at a boundary ( $\approx$ Schwarzschild) time $t$ after the Page time. The state of the radiation then represents the $r \gtrsim r_{z}$ portion of this equal-time hypersurface, where $r_{z}$ is the location of the edge of the zone, and we denote the spacetime point $\left(t, r_{z}\right)$ by $A$; see Fig. 1 . Now, given this radiation state at time $t$, we can determine the state of hard mode excitations that had fallen into the stretched horizon before $t_{\mathrm{w}}$ if $t-t_{\mathrm{w}}$ is of the order of the scrambling time or larger $[10,11]$. (The required $t-t_{\mathrm{w}}$ becomes larger if the amount of information carried by the excitations is large.) Since the black hole information is contained in the soft modes, however, we expect that this time, $t-t_{\mathrm{w}}$, is larger than the signal propagation time between the $r=r_{\mathrm{s}}$ (point $B$ in Fig. 1) and the edge of the zone $r=r_{z}$ (point $A$ ):

$$
t-t_{w} \gtrsim 4 M l_{\mathrm{P}}^{2} \ln \left(M l_{\mathrm{P}}\right)+O\left(M l_{\mathrm{P}}^{2}\right) .
$$

Note that the radiation state at $t$ cannot determine a hard mode excitation at time $t_{\mathrm{w}}$ unless it is hitting the stretched horizon, since the relevant hard modes will not be scrambled by the time $t$. In other words, hard mode operators away from the stretched horizon cannot be represented on the radiation at time $t$.

The fact that hard modes falling before $t_{\mathrm{w}}$ are fully scrambled in the black hole allows us to avoid the energy constraint of the form of Eq. (5) for these modes, as well as the modes entangled with them, and hence to construct corresponding operators of the effective theory at time $t_{\mathrm{w}}$ acting on the state of radiation at time $t$. These operators, however, cannot describe the future of the zone at $t_{\mathrm{w}}$ because hard modes there are not scrambled. A similar 


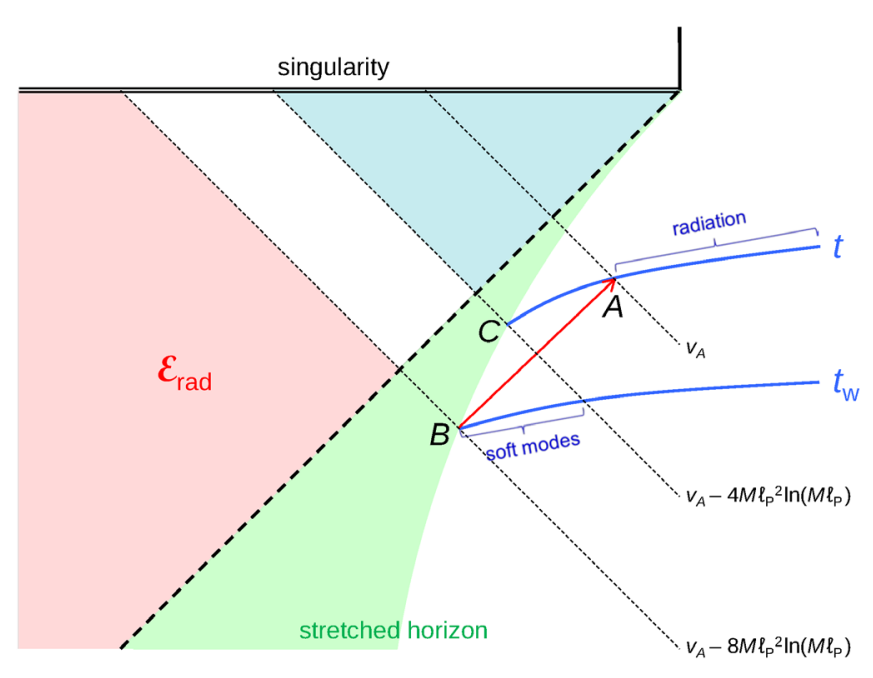

FIG. 1. Radiation of a state given at time $t$ can represent the hard modes hitting the stretched horizon at or before $t_{\mathrm{w}}(<t)$ and the soft modes entangled with them, through time evolution. This allows us to construct the effective interior theory erected at $t_{\mathrm{w}}$ whose operators act only on radiation at $t$; these operators, however, cannot describe the future of the zone at $t_{\mathrm{w}}$, since the radiation cannot represent hard modes there nor the soft modes entangled with them. A similar construction works for effective theories erected at times earlier than $t_{\mathrm{w}}$, implying that we can reconstruct the spacetime region denoted by $\mathcal{E}_{\text {rad }}$, which reproduces the entanglement wedge of the radiation at time $t$. This region, however, cannot describe the fate of a falling object located in the zone at time $t$ (segment $C A$ ), which occurs in the future of $C$ (shaded). To construct an effective theory describing (a part of) this region building on the state at time $t$, we need operators that act both on the soft modes and radiation (in addition to those acting on the hard modes).

construction also works for effective theories before $t_{w}$. This implies that if the scrambling time is as small as the signal propagation time, then we can reconstruct bulk operators in the region denoted by $\mathcal{E}_{\text {rad }}$ in the figure. In $3+1$ dimensions, this indeed reproduces the entanglement wedge of the radiation system given in Ref. [17]. ${ }^{10}$ In $d+1$ dimensions $(d>3)$, the scrambling and signal propagation times are different, and $t-t_{\mathrm{w}}$ must be taken as the scrambling time. We can, however, still check that the scrambling time obtained in Ref. [17] is indeed larger, by a factor of $(d-1) / 2$, than the corresponding signal propagation time between the stretched horizon and the location where Hawking radiation is extracted.

In our view, entanglement wedge reconstruction of the interior using only radiation degrees of freedom describes a collection of (a portion of) spacetime regions associated with effective interior theories that could be erected in the past, specifically at times earlier than the scrambling time before $t$. This leads to some issues (though not necessarily

\footnotetext{
${ }^{10}$ For simplicity, here we have ignored the stretching inside the horizon because it is not relevant for our discussion.
}

problems) from the viewpoint of actually constructing operators describing the interior. First, since the reconstruction involves time evolution backward in time, the expressions for the bulk operators in terms of boundary operators are highly complicated, and the reconstructed operators are extremely fragile; i.e., a small deformation of boundary operators destroys the success of the reconstruction. More importantly, the reconstruction does not provide operators in the interior region that are relevant for describing the fate of an object that is located in the zone region at the time when the state is given.

This can be seen in Fig. 1. In terms of the ingoing Eddington-Finkelstein coordinate $v=t+r_{*}$, where $r^{*}=$ $r+2 M l_{\mathrm{P}}^{2} \ln \left[\left(r-2 M l_{\mathrm{P}}^{2}\right) / 2 M l_{\mathrm{P}}^{2}\right]$ is the tortoise coordinate, point $B$ is $8 M l_{\mathrm{P}}^{2} \ln \left(M l_{\mathrm{P}}\right)$ earlier than point $A$. Namely, the entanglement wedge of the radiation (at time $t$ ) describes only the

$$
v<v_{B}=v_{A}-8 M l_{\mathrm{P}}^{2} \ln \left(M l_{\mathrm{P}}\right)+O\left(M l_{\mathrm{P}}^{2}\right)
$$

portion of the interior spacetime. On the other hand, to describe the future of a falling object that is in the zone at time $t$ (segment $C A$ in the figure), we need the portion

$$
v>v_{C}=v_{A}-4 M l_{\mathrm{P}}^{2} \ln \left(M l_{\mathrm{P}}\right)+O\left(M l_{\mathrm{P}}^{2}\right),
$$

and the two regions in Eqs. (84) and (85) do not overlap. In order to erect an effective theory that is capable of describing future evolution of such an object, we need to use operators that act both on the soft modes and radiation (or, equivalently, on radiation at a sufficiently later time), as discussed in this paper.

\section{CONCLUSIONS AND DISCUSSION}

In this paper, we have shown that operators describing the experience of an observer falling into the horizon can be constructed without contradicting the unitary evolution of the black hole. The choice of these operators at the microscopic level is not unique. In particular, for a young black hole, we can choose them to act only on the degrees of freedom that are directly associated with the black hole: the hard and soft modes. On the other hand, for an old black hole, the operators must also act on radiation emitted earlier. The difference between the two cases comes from the statistics associated with the coarse graining performed to obtain the effective theory of the interior. We have also discussed relation between the present construction and entanglement wedge reconstruction of the interior described in Refs. [17-19].

Before concluding, let us discuss the origin of the coarse graining from a slightly different perspective. From general considerations, we know that the dimension of the Hilbert space describing semiclassical physics in the interior region, $e^{S_{\text {int }}}$, is much smaller than the number of independent black hole or radiation microstates: $S_{\text {int }} \ll S_{\text {bh }} \sim S_{\text {rad }}$ (except 
possibly at the very beginning and end of the black hole evolution). This implies that to erect an effective theory of the interior, we must find very special degrees of freedom within those of the black hole and/or radiation which are relevant for describing the semiclassical physics in the interior spacetime. How can such degrees of freedom be selected?

One way to identify these degrees of freedom is to utilize a subset of semiclassical modes in the exterior of the horizon. The relevant degrees of freedom are then those entangled with these exterior modes, as they allow us to construct operators satisfying the correct annihilation and creation operator algebra up to errors of order $e^{-S_{\text {sys }}}$. This, however, does not necessarily lead to the picture of semiclassical interior spacetime. In particular, for a large AdS black hole we can apply this procedure to exterior modes whose wavelengths are smaller than the horizon size, finding operators that satisfy the correct algebra for each microstate. These operators, however, cannot be extended to linear operators defined throughout the microstates if the frequencies of the modes are smaller than the Hawking temperature. In other words, the Fock spaces built by acting these operators on each microstate significantly overlap with each other.

At first sight, this seems to force us to embrace state dependence of the interior operators in the sense of Refs. [23-25], since it means that the same operator has multiple interpretations. This is, however, not the case if we adopt the view, as we did in this paper, that in a distant description the black hole microstates are represented by the configurations of the soft modes. In this case, exciting a given vacuum microstate by these operators corresponds simply to obtaining other vacuum microstates; a "creation operator" viewed from one vacuum microstate can be viewed as an "annihilation operator" (or a superposition of annihilation and creation operators) from the viewpoint of another vacuum microstate.

By definition, the physics at the semiclassical level should not depend sensitively on the microstate of the black hole or early radiation. This implies that the operators discussed above are not relevant in constructing the theory of the interior at the semiclassical level. The relevant operators are those that act on the modes having frequencies larger than the Hawking temperature - the hard modes - and the degrees of freedom entangled with them. We have shown that the Fock spaces built by these operators on each of the orthogonal vacuum microstates have overlaps exponentially suppressed in the ratio of the energy of semiclassical excitation, $E_{\text {exc }}$, to the Hawking temperature $T_{\mathrm{H}}$. We can therefore define these operators consistently throughout the microstates up to corrections of $e^{-O\left(E_{\text {exc }} / T_{\mathrm{H}}\right)}$. These corrections should be viewed as an intrinsic ambiguity of the semiclassical theory.

We conjecture that the emergence of semiclassical physics, in fact, requires the existence of operators that can be applied globally on a set of microstates with (approximately) invariant meaning, as we have found here. This allows us to view the microscopic Hilbert space as a direct product of the form $\mathcal{H}_{\text {exc }} \otimes \mathcal{H}_{\text {vac }}$ as far as these operators-or observables constructed out of these operators-are concerned. Semiclassical theories are those describing the physics associated with the $\mathcal{H}_{\text {exc }}$ factor, which is insensitive to the microscopic physics occurring in the $\mathcal{H}_{\text {vac }}$ part.

We think this is one of the main lessons we have learned from the paradoxes raised regarding the interior of an evaporating black hole. It is our hope that the picture presented in this paper sheds light on how quantum gravity works at the most fundamental level.

\section{ACKNOWLEDGMENTS}

I would like to thank the organizers and participants of the KITP conference "Geometry from the Quantum"; lively and enjoyable discussions there have motivated me to make a revision integrating the contents of Sec. IV into the main part in a cohesive manner. This work was supported in part by the Department of Energy, Office of Science, Office of High Energy Physics under Contract No. DE-AC0205CH11231 and Grant No. DE-SC0019380, by MEXT KAKENHI Grant No. 15H05895, and by World Premier International Research Center Initiative (WPI Initiative), MEXT, Japan.
[1] J. D. Bekenstein, Black holes and entropy, Phys. Rev. D 7, 2333 (1973).

[2] S. W. Hawking, Particle creation by black holes, Commun. Math. Phys. 43, 199 (1975); Erratum, Commun. Math. Phys. 46, 206 (1976).

[3] S. W. Hawking, Breakdown of predictability in gravitational collapse, Phys. Rev. D 14, 2460 (1976).

[4] L. Susskind, L. Thorlacius, and J. Uglum, The stretched horizon and black hole complementarity, Phys. Rev. D 48, 3743 (1993).
[5] G. 't Hooft, The black hole interpretation of string theory, Nucl. Phys. B335, 138 (1990).

[6] J. Maldacena, The large $\mathrm{N}$ limit of superconformal field theories and supergravity, Int. J. Theor. Phys. 38, 1113 (1999); Adv. Theor. Math. Phys. 2, 231 (1998).

[7] A. Almheiri, D. Marolf, J. Polchinski, and J. Sully, Black holes: Complementarity or firewalls? J. High Energy Phys. 02 (2013) 062.

[8] Y. Nomura, Reanalyzing an evaporating black hole, Phys. Rev. D 99, 086004 (2019). 
[9] Y. Nomura, Spacetime and universal soft modes-Black holes and beyond, Phys. Rev. D 101, 066024 (2020).

[10] P. Hayden and J. Preskill, Black holes as mirrors: Quantum information in random subsystems, J. High Energy Phys. 09 (2007) 120.

[11] Y. Sekino and L. Susskind, Fast scramblers, J. High Energy Phys. 10 (2008) 065.

[12] A. Almheiri, D. Marolf, J. Polchinski, D. Stanford, and J. Sully, An apologia for firewalls, J. High Energy Phys. 09 (2013) 018.

[13] D. Marolf and J. Polchinski, Gauge/Gravity Duality and the Black Hole Interior, Phys. Rev. Lett. 111, 171301 (2013).

[14] R. Bousso, Violations of the Equivalence Principle by a Nonlocally Reconstructed Vacuum at the Black Hole Horizon, Phys. Rev. Lett. 112, 041102 (2014).

[15] D. Marolf and J. Polchinski, Violations of the Born rule in cool state-dependent horizons, J. High Energy Phys. 01 (2016) 008.

[16] G. Penington, S. H. Shenker, D. Stanford, and Z. Yang, Replica wormholes and the black hole interior, arXiv: 1911.11977.

[17] G. Penington, Entanglement wedge reconstruction and the information paradox, arXiv:1905.08255.

[18] A. Almheiri, N. Engelhardt, D. Marolf, and H. Maxfield, The entropy of bulk quantum fields and the entanglement wedge of an evaporating black hole, J. High Energy Phys. 12 (2019) 063.
[19] A. Almheiri, R. Mahajan, J. Maldacena, and Y. Zhao, The Page curve of Hawking radiation from semiclassical geometry, J. High Energy Phys. 03 (2020) 149.

[20] D. N. Page, Information in Black Hole Radiation, Phys. Rev. Lett. 71, 3743 (1993).

[21] W. G. Unruh, Notes on black hole evaporation, Phys. Rev. D 14, 870 (1976).

[22] W. Israel, Thermo field dynamics of black holes, Phys. Lett. 57A, 107 (1976).

[23] K. Papadodimas and S. Raju, An infalling observer in AdS/CFT, J. High Energy Phys. 10 (2013) 212.

[24] K. Papadodimas and S. Raju, State-dependent bulkboundary maps and black hole complementarity, Phys. Rev. D 89, 086010 (2014).

[25] K. Papadodimas and S. Raju, Remarks on the necessity and implications of state-dependence in the black hole interior, Phys. Rev. D 93, 084049 (2016).

[26] J. D. Bekenstein, Universal upper bound on the entropy to energy ratio for bounded systems, Phys. Rev. D 23, 287 (1981).

[27] H. Casini, Relative entropy and the Bekenstein bound, Classical Quantum Gravity 25, 205021 (2008).

[28] I. Kim, E. Tang, and J. Preskill, The ghost in the radiation: Robust encodings of the black hole interior, J. High Energy Phys. 06 (2020) 031.

[29] S. Raju, Smooth causal patches for AdS black holes, Phys. Rev. D 95, 126002 (2017). 